Reprod. Nutr. Dévelop., 1980, 20 (6), 1789-1799.

\title{
Variations saisonnières de la qualité du sperme chez le bélier lle-de-France. I. Etude de la morphologie cellulaire et de la motilité massale $\left(^{*}\right)$
}

\author{
par G. COLAS \\ avec la collaboration technique de Y. GUERIN
}

Station de Physiologie de la Reproduction, I. N. R. A.

Nouzilly, 37380 Monnaie, France.

Summary. Seasonal variations of semen quality in adult lle-de-France rams. I. Study of cell morphology and massal motility of sperm.

Samples were collected (AV) each week $(1 \mathrm{ej} . / 3 /$ week $)$ for 2 years $(1977,1978)$ from 5 adult IF rams of different genetic origins, registered in a flock book. The animals were always kept in a sheepfold. They all received the same feed.

Semen morphology and massal motility were determined for each ejaculate. The percentage of abnormal cells was established by counting 150 cells per smear (1 smear/ô/week; eosin-nigrosin staining). The criteria for celle abnormality were : abnormal head (TA), no tail (SD), proximal droplets (GCP), distal droplets (GCD), abnormal tail (AF).

High ambient temperatures in the shade were recorded during the summer months to study their ultimate effect on semen quality.

Ejaculations were obtained for 2 whole years from all the rams. The main results were as follows.

1. - On the whole, morphological abnormalities (MA) were more frequent from January to June than from July to December : $16.5 \pm 0.9$ vs $13.9 \pm 0.8$ p. $100(P>0.05)$ and $25.3 \pm 1.0$ vs $17.3 \pm 0.9$ p. $100(\mathrm{P}<0.01)$ for 1977 and 1978 , respectively.

The profiles of these abnormalities and their 6-month mean were not very different for the 2 years, except for AF which was nearly always higher in 1978 than in 1977.

The ejaculation usually contained a yearly maximum of abnormal cells from midFebruary up to the end of May (1977 and 1978), indicating that semen quality in this breed closely depends upon the photoperiod.

The maximum points occurred generally in March (4 rams out of 5) but large individual differences between the values were observed $(28$ to 66 p. 100 of MA in $1977 ; 32.7$ to 70.7 in 1978). The length of time during which the proportion of MA was high $(>20$ p. 100) varied considerably with the ram (4 to 16 weeks in 1977, 6 to 17 weeks in 1979). Those differences in animals showed a low or high sensitivity to the increasing photoperiod.

In autumn, on the contrary, the morphological curves were close. An estimate of the reproductive capacity of a sire, based upon morphological examination of the sperm, should thus be made in the spring.

(*) La présente étude sera suivie d'une analyse de la fécondance du sperme et de sa relation avec les paramètres qualitatifs mesurés in vitro (en préparation). 
Temperatures of about $30^{\circ} \mathrm{C}\left(29.1^{\circ} \mathrm{C}\right.$ in $1977 ; 30.8^{\circ} \mathrm{C}$ in 1978) led to an increase in the percentage of TA and SD.

SD and GCP levels for the 2 years were significantly and positively correlated with the total percentage of $M A: 0.63$ and $0.57(P<0.05)$ and 0.77 and $0.74(P<0.01)$ in 1977 and 1978, respectively.

2. - Sperm massal motility varied erratically. The 6-month means were indeed very close (4.3 and 4.2 in $1977 ; 4.0$ and 4.0 in 1978) but the highest variations of the weekly means occurred from October to December, regardless of the year. Thus, motility control cannot be considered as a reliable measurement of quality, especially in the spring.

On connaît avec précision les variations saisonnières de l'activité spermatogénétique chez le bélier (Ortavant, 1958 ; Pelletier, 1971 ; Hochereau-de Reviers, Loir ef Pelletier, 1975). Il est par contre plus difficile de se faire une idée au travers de la littérature, de l'importance des modifications que subit au cours de l'année la qualité du sperme. Plusieurs études ont été consacrées à ce sujet, mais leurs auteurs parviennent à des résultats contradictoires. Les uns (Fowler, 1965) observent des anomalies surtout au printemps, d'autres (Chang, 1941 ; Hafez, Badreldin et Darwish, 1955 ; Smyth et Gordon, 1967 ; El Wishy, El Mikkawi et Omara, 1976) en été (juillet). Enfin, Jennings (1976), Kalev, Zagorski et Sertev (1968) n'enregistrent aucune fluctuation saisonnière sensible dans la qualité du sperme éjaculé. Or, le développement croissant des techniques d'insémination artificielle après induction de l'œstrus (Colas, 1975) conduit à une utilisation quasi permanente des béliers. Il est donc important de savoir si la qualité des gamètes subit ou non une altération au cours de l'année et à quelle époque apparaît cette altération. La présente étude a pour but d'apporter des éléments de réponse à ce problème.

\section{Matériel et méthodes.}

A. Animaux expérimentaux. -5 béliers adultes ( 3 o de 4 ans et 2 de 3 ans) inscrits au livre généalogique de la race lle-de-France et n'ayant entre eux aucun lien de parenté, ont été utilisés au cours des années 1977 et 1978. Les animaux ont vécu en bergerie et reçu tout au long de l'expérience la même ration alimentaire.

B. Recueil et contrôles du sperme. - Les béliers ont été collectés à l'aide d'un vagin artificiel, une fois par semaine (1 seul prélèvement par séance et par bélier) en présence d'une brebis boute-en-train non en chaleur. Aussitôt après la récolte, les éjaculats ont été examinés selon les deux critères suivants :

- motilité massale : notée subjectivement de 0 à 5 (Smyth et Gordon, 1967) après examen $\left(37 \pm 1^{\circ} \mathrm{C}\right)$ au microscope $(\times 80)$;

- anomalies morphologiques.

Pour cet examen, chaque semaine un frottis a été réalisé sur du sperme dilué $[1+1$ à $1+2$ (sperme + dileur) selon la concentration de l'éjaculat] dans une solution de lactose et de jaune d'œuf (Nagase ef Graham, 1964) pour chacun des béliers. Une éłude préliminaire nous avait en effet révélé que la proportion de gout- 
telettes cytoplasmiques distales observée au microscope est significativement plus faible dans le sperme pur que dans le sperme dilué dans un milieu contenant du jaune d'œuf (Colas, résultats non publiés).

La solution de colorant utilisée se composait d'éosine (1 p. 100), de nigrosine (3 p. 100) et de citrate trisodique (3 p. 100) (Ortavant et al., 1953). Son pH était ajusté à 6,7 et sa pression osmotique à 310 milliosmoles. Ces constantes ont été contrôlées régulièrement pendant la durée d'utilisation du colorant (qui n'a jamais excédé un an).

La lecture du frottis (150 cellules/lame) a été réalisée au grossissement $\times 200$. toujours par la même personne. Avant et pendant la numération, les préparations étaient maintenues à environ $37^{\circ} \mathrm{C}$.

Les anomalies ont été classées de la manière suivante :

- têtes anormales (TA) : microcéphales, têtes pyriformes, acrosomes endommagés ;

- têtes sans flagelle (SD) ;

- gouttelettes cytoplasmiques en position proximale (GCP) ;

- gouttelettes cytoplasmiques en position distale (GCD) ;

- anomalies du flagelle (AF) : flagelles courbés, coudés, brisés, pièce principale repliée autour de la pièce intermédiaire ou enroulée à son extrémité.

Lorsqu'un même spermatozoïde présentait deux anomalies (cas peu fréquent), seule l'anomalie la plus grave était recensée selon l'ordre de priorité suivant (TA, SD, GCP, GCD, AF).

Dans tous les cas, le pourcentage total de formes anormales a donc été exprimé à partir de la somme des valeurs relevées dans chaque catégorie.

\section{Expression et analyse des résultats.}

- Expression graphique : L'évolution en fonction de la saison, de la morphologie des gamètes et de la motilité massale du sperme a été exprimée à partir des moyennes hebdomadaires enregistrées sur les cinq béliers.

- Comparaison des moyennes : Les moyennes semestrielles relatives aux anomalies morphologiques ( \pm erreur type) ont été comparées par analyse de variance. Leur calcul a été effectué à partir des mesures individuelles hebdomadaires obtenues au cours des six mois d'observation. Toutefois, pendant le second semestre 1978, deux contrôles (28/9 et $4 / 10)$ n'ont pu être réalisés pour des raisons pratiques. Les moyennes n'ont donc été calculées, dans ce cas, que pour une période de 24 semaines.

Les variations individuelles entre les 5 béliers (différences entre pourcentages maxima ou minima d'anomalies cellulaires) ont été analysées à l'aide du test de Pearson.

Dans le cas de la motilité, les erreurs-types sont indiquées entre parenthèses ef non précédées du signe $t$, les données de base n'ayant pas, par définition, une distribution normale.

- Relevé des tempérafures : Dans le but d'étudier l'incidence éventuelle des fortes températures sur la morphologie cellulaire, nous avons représenté graphiquement les valeurs maxima enregistrées sous abri en 1977 et 1978 pendant la période la plus chaude de l'année (relevés établis par la Station Météorologique de Tours). 
- Corrélation : Les coefficients de corrélation ont été calculés pour chaque année, à partir de moyennes hebdomadaires brutes (et non des pourcentages) obtenues sur les cinq béliers du 15/2 au 31/5 $(n=15)$.

\section{Résultats.}

Les 5 béliers ont pu être collectés régulièrement pendant toute la période expérimentale.

\section{Anomalies morphologiques.}

A. Evolution générale. - L'évolution morphologique des spermatozoïdes collectés sur les 5 béliers pendant la période expérimentale est représentée sur la figure 1 .

Le pourcentage moyen d'anomalies calculé sur six mois est, au cours des deux années, plus élevé de janvier à juin que de juillet à décembre (respectivement $16,5 \pm 0,9$ vs $13,9 \pm 0,8$ p. $100, P>0,05$ en 1977 et $25,3 \pm 1,0$ vs $17,3 \pm 0,9$ p. 100 , $P<0,01$ en 1978).

Le profil d'évolution des TA, SD, GCP et GCD et leur moyenne semestrielle varient peu d'une année à l'autre. Dans le cas des AF, par contre, les moyennes sont significativement plus élevées en 1978 qu'en 1977 (6,6 $\pm 0,6$ vs 13,5 \pm 0,9 p. 100, $P<0,01$ et $7,8 \pm 0,4$ vs $10,7 \pm 0,7$ p. 100, $P<0,01$ respectivement pour les deux premiers semestres de chaque année). Cette augmentation explique que la proportion d'anomalies totales soit plus élevée en 1978 qu'en 1977.

Au cours du premier semestre, c'est en mars que l'on rencontre le plus fort taux de TA ( 8,0 p. 100 en $1977 ; 8,4$ p. 100 en 1978). De juillet à décembre, abstraction faite d'un pic au mois de juillet 1978 (vraisemblablement dû aux effets d'un traitement par les antibiotiques pratiqué sur un bélier quatre semaines plus tôt), les valeurs les plus hautes apparaissent en août (5,7 p. 100 en 1977 ; 4,4 p. 100 en 1978), c'est-à-dire un mois environ après les plus fortes chaleurs.

La présence de GCP, limitée principalement aux mois de février, mars et avril, est maximum en mars (3,2 p. 100 en $1977 ; 3,5$ p. 100 en 1978). On l'observe chez tous les béliers à cette époque mais avec une fréquence et une intensité variables selon les animaux.

Les SD augmentent en février et en juillet pour atteindre un maximum en mars et en août (resp. 5,2 et 6,7 p. 100 en $1977 ; 8,4$ et 5,6 p. 100 en 1978). La remontée importante observée au mois d'août semble être à nouveau la conséquence de très fortes températures relevées sous abri le mois précédent $\left(29,1^{\circ} \mathrm{C}\right.$ en $1977,30,8^{\circ} \mathrm{C}$ en 1978).

Les GCD s'observent toute l'année et en bien plus grand nombre que les GCP. Néanmoins, elles sont toujours significativement plus abondantes au cours des six premiers mois de l'année.

Les malformations des flagelles atteignent leur maximum au printemps (12,5 p. 100 en mars $1977 ; 21,9$ p. 100 en avril 1978). Il faut toutefois préciser qu'à cette époque (mars à mai) les altérations (flagelles sans pièce principale ou enroulés autour de la pièce intermédiaire) sont généralement plus graves qu'en automne (flagelles coudés ou courbés). 


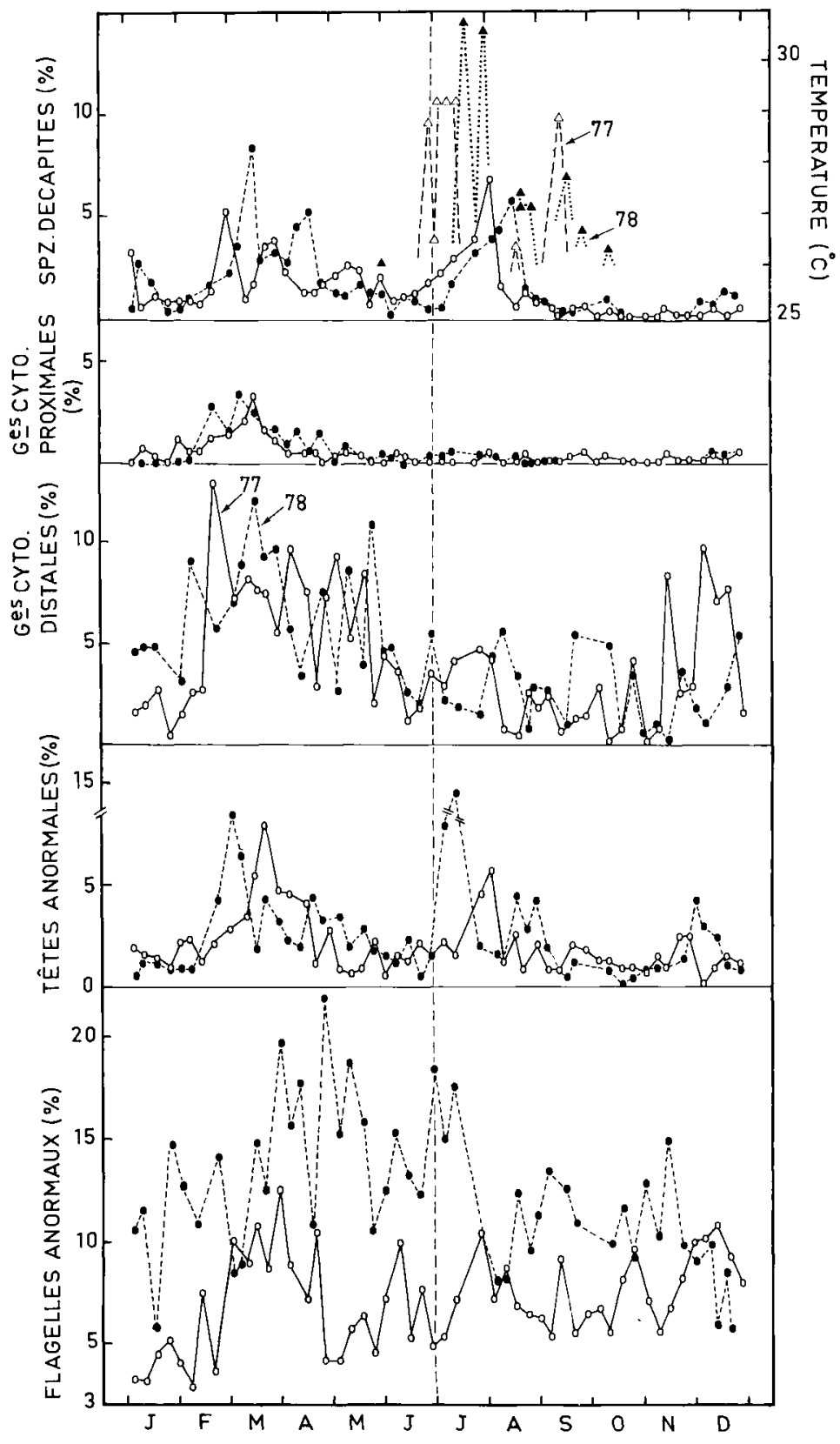

FIG. 1. - Evolution hebdomadaire des anomalies morphologiques (०-०) (•- -๑) du sperme de bélier llede-France et température journolières maxima $(\Delta--\Delta, \Delta, \ldots \Delta)$ au cours des années $1977(\Delta)$ ef 1978 (৯) $(n=5)$. 
B. Différences individuelles. - Jusqu'au 15 juin (photopériode croissante) la durée pendant laquelle les pourcentages d'altérations morphologiques sont supérieurs à 20 p. 100 (fig. 2) varie selon les animaux : 4 à 16 semaines en 1977, 6 à 17 semaines en 1978.

1977

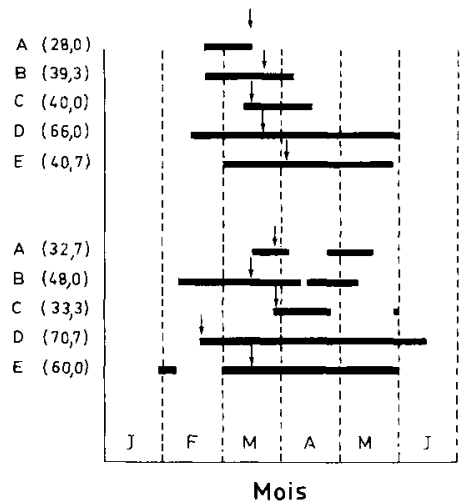

FIG. 2. - Apparition ef durée des périodes à fort pourcentage d'anomalies (> 20 p. 100), en liaison ovec la photopériode croissante, chez le bélier lle-de-Fronce $(n=5)$. Les flèches indiquent les pourcentages maxima ( ) observés.

Les valeurs maxima sont significaiıvement très différentes $(28,0$ à 66,0 p. 100 en $1977, \mathrm{P}<0,01 ; 32,7$ à 70,7 p. 100 en $1978, \mathrm{P}<0,01$ ).

Quatre fois sur cinq les maxima s'observent au mois de mars. D'une année à l'autre, ef pour chaque animal, il y a peu de changement dans le moment d'apparition de ces valeurs, sauf pour le bélier $D$ (qui présentait tout de même 66 p. 100 de spermatozoïdes anormaux le 20.3.1978).

On voit également que les 2 béliers qui ont le profil morphologique le plus mauvais ( $D$ et $E$ ) en 1977, restent aussi les deux plu; inal classés en 1978.

En automne, au contraire, les profils morphologiques des 5 béliers diffèrent peu et les pourcentages minima d'AM sont voisins : 5,3 à 6,7 p. 100 en 1977, 4,7 à 6,7 p. 100 en 1978.

C. Corrélation entre anomalies morphologiques. - Les corrélations entre les différentes formes d'anomalies ont été regroupées dans le tableau 1. Pour chaque coefficient les deux valeurs obtenues (1977 et 1978) sont souvent de signe opposé. Cela s'observe surtout dans le cas des AF. Les SD et les GCP sont les seules malformations à être corrélées positivement et de manière significative, au cours des deux années, aux anomalies totales $(0,63$ et 0,57 en 1977 et 1978 et 0,77 et 0,74 pour SD et GCP).

\section{Motilité massale.}

La motilité massale du sperme pur évolue chaque année d'une façon assez aléatoire et différente de celle de la morphologie cellulaire (fig. 3). C'est au cours du secorid semestre et plus précisément d'octobre à décembre que les moyennes hebdomadaires varient le plus (3,0 à 4,8 en 1977, 2,4 à 4,5 en 1978).

Les moyennes semestrielles sont très voisines : 
TABLEAU 1

Corrélations statistiques entre anomalies morphologiques (calculées entre le $15 / 2$ et le $31 / 5, n=15,1977$ et 1978)

\begin{tabular}{|c|c|c|c|c|c|}
\hline \multirow{2}{*}{ Anomalies } & \multicolumn{5}{|c|}{ Anomalies morphologiques } \\
\hline & AF & SD & GCP & GCD & TA \\
\hline AF & & & & & \\
\hline SD .. & $\begin{array}{r}0,50 \\
-0,08\end{array}$ & & & & \\
\hline GCP $\ldots \ldots \ldots$ & $\begin{aligned} & 0,52 * \\
- & 0,08\end{aligned}$ & $\begin{array}{l}0,31 \\
0,44\end{array}$ & & & \\
\hline GCD $\ldots \ldots \ldots$ & $\begin{array}{r}-0,24 \\
0,09\end{array}$ & $\begin{array}{l}0,12 \\
0,35\end{array}$ & $\begin{array}{l}0,29 \\
0,40\end{array}$ & & \\
\hline TA $\ldots . . .$. & $\begin{array}{r}0,50 \\
-0,57 *\end{array}$ & $\begin{array}{r}0,36 \\
-0,34\end{array}$ & $\begin{array}{l}0,63^{*} \\
0,42\end{array}$ & $\begin{array}{l}0,20 \\
0,02\end{array}$ & \\
\hline Totales ...... & $\begin{array}{l}0,68 * * \\
0,26\end{array}$ & $\begin{array}{l}0.63^{*} \\
0.57^{*}\end{array}$ & $\begin{array}{l}0,77^{* *} \\
0,74 * *\end{array}$ & $\begin{array}{l}0,46 \\
0,65 * *\end{array}$ & $\begin{array}{l}0,80 * * \\
0,21\end{array}$ \\
\hline
\end{tabular}

*: $\mathrm{P}<0,05 ; * *: \mathrm{P}<0,01$.

Pour chaque corrélation, les deux valeurs indiquées correspondent aux années 1977 ef 1978. AF : anomalie du flagelle; $S D$ : spz décapité ; CGP : Gouttelette cytoplasmique proximale ; $G C D$ : gouttelette cytoplasmique distale; TA : tête anormale.

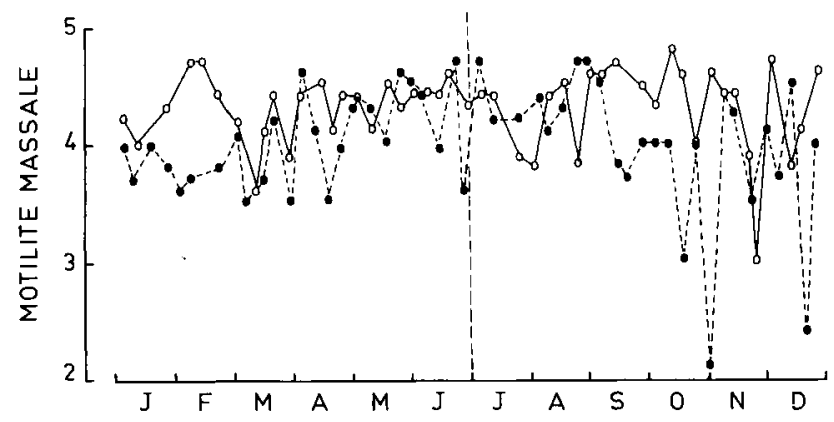

FIG. 3. - Evolution hebdomodoire de la motilité du sperme de bélier lle-de-France au cours des années $1977(\circ-\circ)$ ef $1978(\bullet-\bullet)(n=5)$

\section{Discussion.}

L.es résultats que nous venons de présenter montrent que la qualité du sperme des béliers lle-de-France varie beaucoup au cours de l'année.

1. Action de la photopériode.

A. Sur la morphologie cellulaire. - Si l'on exclut les malformations du flagelle qui semblent varier d'une façon assez arbitraire, on voit que la proportion d'anomalies est élevée de la mi-février à la fin ma et qu'elle atteint son maximum au mois de mars chez la plupart des béliers. Le phénomène se répète d'une année sur l'autre. Or, pendant cette période, la lumière est seule à agir, au moins sous nos latitudes. 
L'augmentation de la durée du jour entraine donc l'apparition dans le sperme de nombreuses anomalies de forme. Son effet est d'ailleurs très marqué puisqu'à certaines époques, il s'exerce sur plus du tiers des gamètes. On savait déjà que la semence des béliers lle-de-France reflète, dans sa qualité, les fluctuations d'un éclairement artificiel (Colas, 1979). Il n'est donc pas étonnant d'enregistrer en milieu naturel également, des réponses très différentes entre printemps et automne. On sait en outre (Ortavant, 1958) que la lumière exerce aussi une action prépondérante sur la spermatogenèse chez l'lle-de-France. Cela nous incite à penser que toute race dont l'activité testiculaire est fortement influencée par la photopériode doit, de même, subir des variations saisonnières très marquées dans la qualité des éjaculats produits. D'autres données (non publiées ici), recueillies à l'occasion de sondages effectués sur deux génotypes différents du point de vue de la longueur de la saison sexuelle (Texel et Préalpes), étayent cette supposition.

Le profil morphologique observé à cette époque de l'année peut être considéré comme caractéristique de la race lle-de-France. Chaque race doit ainsi avoir une "période critique » que l'on pourrait définir par sa durée et par son pourcentage moyen d'altérations cellulaires.

Les différences individuelles dans les proportions d'anomalies sont faibles en automne mais très importantes au printemps. C'est donc au moment où la durée d'éclairement augmente et en particulier de la mi-février à la fin mai qu'il faut juger la valeur des reproducteurs et cela d'autant plus que l'on s'oriente maintenant dans la pratique vers une utilisation des mâles à contre-saison. Ce jugement peut être fondé sur le contrôle de l'évolution morphologique du sperme par le test que nous avons utilisé et dont l'emploi est simple et rapide.

Les gouttelettes cytoplasmiques proximales n'apparaissent dans l'éjaculat qu'en lumière croissante et toujours aux mêmes mois de l'année: Elles sont donc spécifiques de l'action de la lumière et traduisent une certaine perturbation des phénomènes de maturation dans l'épididyme, comme l'avaient déjà pressenti Fournier-Delpech ef al. (1979). De plus, ce sont les malformations les mieux corrélées, pendant deux années consécutives, au pourcentage total de cellules anormales. Leur présence, en proportion plus ou moins élevée dans le sperme, traduit une sensibilité plus ou moins forte de l'animal vis-à-vis de la photopériode.

Comme Hafez et al. (1955), Smith et Gordon (1967), nous trouvons que les anomalies du flagelle sont très fréquentes chez le bélier. En première analyse, leur évolution ne paraît pas liée étroitement à celle de la lumière. Toutefois, comme nous l'avons signalé, les altérations observées sont en général de nature différente au printemps et à l'automne. Une analyse plus détaillée du phénomène aurait sans doute permis de nuancer notre résultat.

B. Action sur la motilité massale. - La motilité initiale du sperme n'est pas plus élevée en automne qu'au printemps. Un résultat comparable avait déjà été obtenu par Colas et Courot (1976) sur les béliers Ile-de-France mainfenus sous éclairement artificiel. Les fluctuations photopériodiques n'ont donc pas d'effet sensible sur la motilité massale chez cette race et sans doute pas non plus chez d'autres races européennes comme le laissent penser les travaux de Starke (1949), Gordon (1958), Smyth el Gordon (1967). 
Dans les conditions où elle est généralement observée, la motilité reste, de toute manière, une donnée subjective et imprécise qui n'est reliée étroitement ni à la durée d'éclairement, ni au pouvoir fécondant (Colas ef Courot, 1977). Sa mesure ne traduit selon Hafez ef al. (1955) qu'une plus ou moins forte proportion de cellules vivantes. En période de faible activité sexuelle, lorsque la semence est riche en anomalies cellulaires, l'examen de la turbulence d'un éjaculat ne saurait donc être qu'un complément du bilan morphologique qui, lui, est beaucoup plus fiable.

\section{Température.}

Les températures maxima que nous avons enregistrées sont très au-dessous des valeurs généralement considérées comme pouvant perturber la fonction de reproduction chez le bélier (Dutt et Hamm, 1957 ; Lindsay, 1965 ; Waites et Ortavant, 1968 ; Rathore et Yeates, 1967 ; Rathore, 1968, 1969 ; Zaba-Branny, 1971). Pourtant, nous avons pu voir à deux reprises (1977 et 1978) que leur action, tout en étant bien dissociée de celle de la lumière, est loin d'être négligeable. Il suffit que les animaux soient exposés quelques heures à $29^{\circ} \mathrm{C}$ pendant 3 jours ou à $30^{\circ} \mathrm{C}$ pendant 2 jours consécutifs pour que la proportion de spermatotozoïdes anormaux augmente. Par contre, ces températures ne sont suivies d'aucun effet lorsqu'elles ne durent qu'un seul jour (1re quinzaine de septembre 1977). La température minimum susceptible d'entraîner des altérations morphologiques dans la semence des béliers lle-de-France se situe donc aux environs de $30^{\circ} \mathrm{C}$. Encore faut-il, pour qu'elle agisse, qu'elle se renouvelle pendant plusieurs jours de suite. Ce seuil varie selon le génotype comme l'a montré Lindsay (1965). De ce point de vue, la race lle-de-France peut donc être considérée comme assez thermosensible.

L'effet maximum de la température sur le sperme n'apparaît qu'aụ bout d'un mois environ, ce qui confirme les résultats de Dutt ef Hamm (1957). Il se traduit par une augmentation du pourcentage de spermatozoïdes décapités et d'anomalies de la tête, comme l'avaient déjà signalé plusieurs auteurs (Dutt et Hamm, 1957 ; Rathore ef Yeates, 1967 ; Rathore, 1968).

\section{Conclusion.}

Chez le bélier lle-de-France, la morphologie des spermatozoïdes subit des modifications profondes au cours de l'année sous l'effet de la photopériode croissante (mi-février à fin mai). Sous nos climats, la température agit également (août) mais à un degré beaucoup moindre.

C'est au mois de mars que l'altération est la plus forte, mais il existe des différences individuelles importantes dans l'intensité et la durée du phénomène. De tels écarts permettent d'envisager un tri des béliers sur leur sensibilité au photopériodisme.

En revanche, la motilité massale du sperme varie sans liaison apparente avec ces conditions de milieu. 
Remerciements. - Nous tenons à exprimer nos remerciements à Mlle Solari ef à M. Mariana pour leurs conseils dans l'interprétation statistique des résultats et à $M$. Courot pour ses commentaires sur la présente étude.

\section{Références}

CHANG M. C., 1941. A study of the physiology of ram spermatozoa. Thesis Ph. D. Cambridge Univ., England.

COLAS G., 1975. The use of progestagen SC 9880 as an aid for artificial insemination in the sheep. Ann. Biol. anim. Bioch. Biophys., 15, 317-327.

COLAS G., COUROT M., 1977. Production of spermatozoa, storage of semen and A. I. in the sheep. Proc. Symp. Management of reproduction in sheep and goats, 24 July, Wisconsin, 31-40.

COLAS G., 1979. Fertility in the ewe after artificial insemination with fresh and frozen semen at the induced cestrus and influence of the photoperiod on the semen quality of the ram. Livest. Prod. Sci., 6, 153-166.

COLAS G., GUERIN Y., 1979. L'insémination artificielle chez les ovins : acquisitions ef perspectives. 5 e journ. Rech. Ovine et Caprine. "L'amélioration généfique des espèces ovine et caprine", ITOVIC éd. Paris, 162-185.

DUTT R. H., HAMM P. T., 1957. Effect of exposure to high environmental temperature and shearing on semen production of rams in winter. J. anim. Sci., 16, 328-334.

EL-WISHY A. B., EL MIKKAWI F., OMARA A., 1976. Some aspects of reproduction in fat-tailed sheep in the subtropics. $V$. Seasonal variations in sexual desire and semen characteristics. Beitr. Tropisch. Landwirsich. Veterinärmed., 14, 303-310.

FOURNIER-DELPECH S., COLAS G., COUROT M., BRICE G., 1979. Epididymal sperm maturation in the ram : motility, fertilizing ability and embryonic survival after uterine artificial insemination in the ewe. Ann. Biol. anim. Bioch. Biophys., 19, 597-605.

FOWLER D. G., 1965. Semen quality of Merino rams. 2. The effects of seasonal changes in daylength on semen quality. Aust. J. exp. Agric. anim. Husb., 5, 247-251.

GORDON I., 1958. The use of progesterone and serum gonadotrophin (PMS) in the control of fertility in sheep. II. Studies in the extra-seasonal production of lambs. J. agric. Sci., 50, 152-197.

HAFEZ E. S. E., BADRELDIN A. L., DARWISH Y. H., 1955. Seasonal variations in semen characteristics of sheep in the subtropics. J. agric. Sci., 45, 283-292.

HOCHEREAU-de REVIERS M. T., LOIR M., PELLETIER J., 1975. Seasonal variations in the response of the testis and LH levels to hemicastration of adult rams. J. Reprod. Fert., 46, 203-209.

JENNINGS J. J., 1976. Effect of season and mating frequency on semen characteristics in rams. VIIlth Int. Congr. onim. Reprod. artif. Insem., Krakow, IV, 998-1001.

KALEV G., ZAGORSKI D., SERTEV M., 1968. Sur l'intensification de la production de spermatozoïdes chez les béliers. VIe Congr. int. Reprod. anim. Insém., artif., Paris, Vol. I, 289-291.

LINDSAY D. R., 1965. Sexual activity and semen production of rams at high temperatures. J. Reprod. Fert., 18, 1-8.

NAGASE N., GRAHAM E. F., 1964. Pelleted semen : comparison of different extenders and processes on fertility of bovine spermatozoa. Proc. $5^{\text {th }}$ int. Congr. Anim. Reprod. artif. Insem., Trento, 2, 387-389.

ORTAVANT R., DUPONT G., PAUTHE H., ROUSSEL G., 1953. Contribution à l'élude de la différenciation des spermatozoïdes morts et des spermatozoïdes vivants dans le sperme de taureau. Ann. Zootech., 2, 1-8.

ORTAVANT R., 1958. Le cycle spermatogénétique chez le bélier. Th. Doct. Sci. Paris, 127 pp., No A 3118-0 3990.

PELLETIER J., 1971. Influence du photopériodisme el des androgènes sur la synthèse et la libération de LH chez le bélier. Th. Doct. Sci. Paris, 243 Pp., no 105441.

RATHORE A. K., 1968. Effects of high temperature on sperm morphology and subsequent fertility in merino sheep. Proc. Austr. Soc. onim. Prod., 7, 270-274.

RATHORE A. K., 1969. Mid-piece sperm abnormality due to high femperature exposure of rams. Br. Vet. J., 125, 534-538. 
RATHORE A. K., YEATES N. T. M., 1967. Morphological changes in ram spermatozoa due to heat stress. Vet. Rec., 81, 343-345.

SMYTH P., GORDON I., 1967. Seasonal and breed variations in the semen characteristics of rams in Ireland. Irish Vet. J., 21, 222-233.

STARKE N. C., 1949. The sperm picture of rams of different breeds as an indication of their fertility. II. The rate of sperm travel in the genital tract of the ewe. Onderstepoort J. vet. Sci., 22, 415-425.

WAITES G. M. H., ORTAVANT R., 1968. Effets précoces d'une brève élévation de la température testiculaire sur la spermatogenèse du bélier. Ann. Biol. anim. Bioch. Biophys., 8, 323-331.

ZABA-BRANNY A., 1971. Effect of exposure to high environmental temperature on production and characteristics of semen in the ram. Acta Agr. silvestr., 11, 69-92. 